\title{
Hypoxia induces glucose uptake and metabolism of adipose-derived stem cells
}

\author{
HYOUNG SOOK PARK ${ }^{1}$, JI HYE KIM ${ }^{1}$, BO KYUNG SUN ${ }^{1}$, SUN U. SONG ${ }^{2}$, \\ WONHEE SUH ${ }^{3}$ and JONG-HYUK SUNG ${ }^{1,4}$ \\ ${ }^{1}$ Department of Pharmaceutics and Institute of Pharmaceutical Science, College of Pharmacy, Yonsei University, \\ Incheon 21983; ${ }^{2}$ Translational Research Center, Inha University School of Medicine, Incheon 22332; \\ ${ }^{3}$ Department of Pharmacy, College of Pharmacy, Chung-Ang University, Seoul 06974; \\ ${ }^{4}$ Stemore Co. Ltd., Incheon 21983, Republic of Korea
}

Received September 25, 2015; Accepted September 12, 2016

DOI: $10.3892 / \mathrm{mmr} .2016 .5796$

\begin{abstract}
It has previously been demonstrated that hypoxia has diverse stimulatory effects on adipose-derived stem cells (ASCs), however, metabolic responses under hypoxia remain to be elucidated. Thus, the present study aimed to investigate the glucose uptake and metabolism of ASCs under hypoxic conditions, and to identify the underlying molecular mechanisms. ASCs were cultured in $1 \%$ oxygen, and experiments were conducted in vitro. As determined by proteomic analysis and western blotting, GAPDH and enolase 1 (ENO1) expression were upregulated under hypoxia. In addition, lactate production was significantly increased, and mRNA levels of glycolytic enzymes, including GAPDH, ENO1, hexokinase 2 (HK2), and lactate dehydrogenase $\alpha(\mathrm{LDH} \alpha)$ were upregulated. Hypoxia-inducible factor 1- $\alpha$ (HIF-1 $\alpha)$ expression was increased as demonstrated by western blotting, and a pharmacological inhibitor of HIF-1 $\alpha$ significantly attenuated hypoxia-induced lactate production and expression of glycolytic enzymes. It was also observed that hypoxia significantly increased glucose uptake in ASCs, and glucose transporter (GLUT)1 and GLUT3 expression were upregulated under hypoxia. Pharmacological inhibition of the HIF-1 $\alpha$ signaling pathways also attenuated hypoxia-induced GLUT1 and GLUT3 expression. These results collectively indicate that hypoxia increases glucose uptake via GLUT1 and GLUT3
\end{abstract}

Correspondence to: Professor Jong-Hyuk Sung, Department of Pharmaceutics and Institute of Pharmaceutical Science, College of Pharmacy, Yonsei University, 162-1 Songdo-dong, Yeonsu-gu, Incheon 21983, Republic of Korea

E-mail: brian99@empal.com

Professor Wonhee Suh, Department of Pharmacy, College of Pharmacy, Chung-Ang University, 84 Heukseok-Ro, Dongjak-Gu, Seoul 06974, Republic of Korea

E-mail:wsuh@cau.ac.kr

Key words: hypoxia, adipose-derived stem cells, lactate production, glucose uptake, proliferation upregulation, and induces lactate production of ASCs via GAPDH, ENO1, HK2, and LDH $\alpha$. Furthermore, HIF-1 $\alpha$ is involved in glucose uptake and metabolism of ASCs.

\section{Introduction}

It has previously been demonstrated that hypoxia has diverse effects on human adipose-derived stem cells (ASCs). For example, $2 \%$ hypoxia increases the proliferation and migration of ASCs via reactive oxygen species (ROS) generation (1-3). ROS are primarily generated by NADPH oxidase in ASCs, which mediates proliferation and migration (4). Hypoxia-generated ROS increase phosphorylation of platelet-derived growth factor receptor- $\beta$, and activation signaling is followed by Akt and extracellular-regulated kinase (ERK) phosphorylation to increase the proliferation and migration of ASCs (2,5-7). Activation of Akt and ERK pathways induces nuclear factor- $\kappa \mathrm{B}$ and ETS domain-containing protein Elk-1 phosphorylation to upregulate miR-210 (termed 'hypoxamir') expression, which downregulates protein tyrosine phosphatase, non-receptor type 2 to increase the proliferation and migration of ASCs (8). ROS generation by rotenone and antimycine was demonstrated to upregulate miR-210 expression, which resulted in miR-210 being termed 'ROSmir' (8). In addition, hypoxia markedly increases the secretion of vascular endothelial growth factor, hepatocyte growth factor, and basic fibroblast growth factor (bFGF) from ASCs, which collectively enhance the wound-healing and hair-regenerative potential of ASCs $(3,9)$. Hypoxia also has effects on ASC transdifferentiation. Hypoxia generates ROS and mediates adipocyte differentiation of ASCs (10). Hypoxia generally increases chondrogenic differentiation while inhibiting the osteogenic differentiation of ASCs $(11,12)$. As described above, alteration of mitogenic and paracrine effects of ASCs under hypoxia have been well-studied, however, the effects on glucose utilization and metabolism during hypoxia in ASCs requires further elucidation.

Glycolysis is the metabolic pathway that converts glucose into pyruvate, and the free energy released in this process is used to form the high-energy compounds, adenosine triphosphate and NADH. Glycolysis occurs in aerobic and anaerobic conditions, and anaerobic metabolism has been reported to be 
beneficial to maintain the self-renewal of stem cells $(13,14)$. Stem cells heavily rely on anaerobic glycolysis to increase survival and proliferation, and stem cell function is also regulated by bioenergetic signaling pathways, such as the AMP-activated protein kinase (AMPK) and hypoxia inducible factor (HIF) pathways $(14,15)$. In addition, differentiation is accompanied by a shift from anaerobic glycolysis to mitochondrial respiration, and this metabolic switch of differentiating stem cells is required to cover the energy demands of organ-specific differentiating cells (13). It is reasonable to assume that there is an association between the self-renewal state of stem cells and the activity of specific metabolic pathways (16).

ASCs exist in hypoxic microenvironments of adipose tissue, and ASCs cultured under hypoxia ex vivo (1-5\% oxygen) reportedly have advantages in proliferation, migration and growth factor secretion over those cultured under normoxia $(1,5,17,18)$. Anaerobic metabolism under hypoxia during ex vivo expansion may be beneficial to ASC self-renewal and proliferation, however, altered glucose uptake and metabolism of ASCs under hypoxia has not been fully characterized. Thus, the present study investigated the altered glucose uptake and lactate production of ASCs under hypoxia (1\% oxygen). In addition, the current study aimed to identify the molecular mechanisms involved in hypoxia-induced glucose uptake and lactate production by ASCs.

\section{Materials and methods}

Materials and antibodies. Deferoxamine (DFX; a hypoxia-mimic agent), rapamycin (a mammalian target of rapamycin inhibitor), and sodium oxamate [a lactate dehydrogenase (LDH) inhibitor] were purchased from Sigma-Aldrich (Merck Millipore). Compound C (an AMPK inhibitor) was purchased from EMD Millipore (Billerica, MA, USA). YC-1 (a HIF-1 $\alpha$ inhibitor) was purchased from AG Scientific Inc. (San Diego, CA, USA). GAPDH and tubulin were purchased from Santa Cruz Biotechnology, Inc. (Dallas, TX, USA). An antibody against enolase 1 (ENO1; cat. no. 11204-1-AP) was purchased from Proteintech Group, Inc. (Rosemont, IL, USA). An antibody against HIF-1 $\alpha$ (cat. no. 610958) was purchased from BD Biosciences (San Jose, CA, USA). Antibodies against glucose transporter (GLUT)1 (cat. no. ab652) and GLUT3 (cat. no. ab15311) were purchased from Abcam (Cambridge, UK).

Cell culture. ASCs were isolated from lipoaspirates of human subcutaneous adipose tissue from two females in 2011 and characterized by flow cytometry using selected cell surface makers as described previously (19). Informed consent was obtained from patients at Bundang CHA Hospital (Sungnam, Korea), and approved by the ethics committee of Chung-Ang University (no. BD2011-152D; Pocheon, Korea). ASCs were characterized using cell surface markers, including cluster of differentiation (CD)34, CD73, CD90, and CD105, and multiple differentiation states were identified (19). ASCs were cultured in $\alpha$-minimum essential medium ( $\alpha$-MEM; Hyclone; GE Healthcare Life Sciences, Logan, UT, USA) with $10 \%$ fetal bovine serum (Gibco; Thermo Fisher Scientific, Inc.) and $1 \%$ penicillin (Gibco; Thermo Fisher Scientific, Inc.). ASCs were incubated in $5 \% \mathrm{CO}_{2}$ and $20 \% \mathrm{O}_{2}$ (normoxia) or
$1 \% \mathrm{O}_{2}$ (hypoxia) with the same percentage $\mathrm{N}_{2}$, at $37^{\circ} \mathrm{C}$. ASCs at passages 4-7 were used in the current study.

Two-dimensional (2D) electrophoresis and protein identification by mass spectrometry. ASCs under normoxia and hypoxia were used for 2D electrophoresis. Protein was obtained by scraping in SDS lysis buffer. Extracts were incubated on ice for $30 \mathrm{~min}$ and supernatant was harvested by centrifugation at $13,400 \times \mathrm{g}$ for $10 \mathrm{~min}$. Subsequently, quantification of protein was measured by Bradford assay. To analyze in the first dimension, $1 \mathrm{mg}$ protein was electrofocused on immobilized $\mathrm{pH}$ 3-10 nonlinear $\mathrm{pH}$ gradient strip (GE Healthcare Life Sciences, Chalfont, UK). For separation in the second dimension, isoelectrically focused strips were electrophoresed on gradient polyacrylamide gels. The relative abundance of protein spots was quantified by staining the gels with Coomassie Brilliant Blue followed by scanning with a GS-710 imaging densitometer (Bio-Rad Laboratories, Inc., Hercules, CA, USA) and analysis with Melanie 7 image analysis software (GE Healthcare Life Sciences). Labeled images were analyzed using Adobe Photoshop (version 7.0) software (Adobe Systems, Inc., San Jose, CA, USA). For matrix-assisted laser desorption ionization-time of flight (MALDI-TOF) analysis, tryptic peptides were desalted and purified using a mixture of Poros R2 and Oligo R3 (Applied Biosystems; Thermo Fisher Scientific, Inc.). The mass spectrometry (MS) spectra of peptides were determined by spectrometric analysis

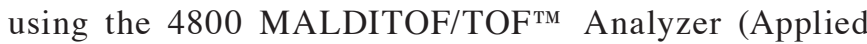
Biosystems; Thermo Fisher Scientific, Inc.). The operating software used was Applied Biosystems 4000 series Data Explorer version 4.4. At the end of the macro process, the centroid mass, resolution, height, and signal/noise ratio were determined for each peak. Data were converted to a Microsoft Excel file (Microsoft Corporation, Redmond, WA, USA) and used for a Mascot search (Matrix Science, Ltd., London, UK; version 2.2.04). For LC-MS/MS analysis, a nano chip column (150x0.075 mm; Agilent Technologies, Inc., Santa Clara, CA, USA) was used for peptide separation. Ion spectra were determined in the information-dependent acquisition mode and analyzed by Agilent 6530 Accurate-Mass Q-TOF LC-MS system (Agilent Technologies, Inc.). Mascot (version 2.2.04) was used to identify peptide sequences present in the protein sequence database of the National Center for Biotechnology Information (human).

Western blotting. Proteins were isolated with SDS lysis buffer and quantified using a Bradford assay. Lysates $(30 \mu \mathrm{g})$ were separated in $8 \%$ or $10 \%$ SDS-polyacrylamide gels and transferred to Immobilon-P membranes (EMD Millipore). Membranes were blocked with 5\% skim milk in Tris-buffered saline containing $0.2 \%$ Tween 20 for $30 \mathrm{~min}$ at room temperature and incubated overnight with primary antibodies diluted in blocking solution at $4^{\circ} \mathrm{C}$. Primary antibodies used were anti-ENO (1:1,000; anti-rabbit IgG; Proteintech Group, Inc.), HIF-1 $\alpha$ (1:1,000; anti-mouse IgG1), GLUT1 (1:1,000; anti-rabbit IgG), GLUT3 (1:1,000; anti-rabbit IgG), GAPDH (1:3,000; anti-mouse IgG; cat. no. sc-47724; Santa Cruz Biotechnology, Inc.) and tubulin (1:3,000; anti-mouse IgG; cat. no. sc-5274; Santa Cruz Biotechnology, Inc.). The membranes were incubated with a horseradish peroxidase 
(HRP)-conjugated secondary antibody (1:10,000; anti-mouse IgG, cat. no. PI-2000; anti-rabbit IgG, cat. no. PI-1000; Vector Laboratories, Ltd., Peterborough, UK) for $1 \mathrm{~h}$ in blocking solution, and blots were visualized using Immobilon Western Chemiuminescent HRP Substrate (EMD Millipore).

$R N A$ isolation and reverse transcription-quantitative polyerase chain reaction ( $R T-q P C R)$. Total RNA was isolated from ASCs using an RNeasy Mini kit (Qiagen GmbH, Hilden, Germany) and cDNA was synthesized using a cDNA synthesis kit (Promega Corporation, Madison, WI, USA). The GLUT1, GLUT3, ENO1, GAPDH, hexokinase 2 (HK2), LDH $\alpha$ and 18S rRNA cDNA were amplified using SYBR Green PCR Master mix (Takara Bio, Inc., Otsu, Japan) in a StepOne ${ }^{\mathrm{TM}}$ Real-time PCR system (Applied Biosystems; Thermo Fisher Scientific, Inc.). PCR reactions were incubated for $2 \mathrm{~min}$ at $95^{\circ} \mathrm{C}$, followed by 35 cycles of $20 \mathrm{sec}$ at $95^{\circ} \mathrm{C}, 40 \mathrm{sec}$ at $58^{\circ} \mathrm{C}$ and $1 \mathrm{~min}$ at $72^{\circ} \mathrm{C}$, with a final step of $5 \mathrm{~min}$ at $72^{\circ} \mathrm{C}$. The quantity of each cDNA was normalized by dividing it by the $18 \mathrm{~S}$ rRNA level in the corresponding sample. The sequences of the qPCR primers were as follows: Forward, 5'-TCACTGTGC TCCTGGTTCTG-3' and reverse, 5'-CTTGTGCTCCTGAGA GATCC-3' for GLUT1; forward, 5'-ACCGGCTTCCTCATT ACCTT-3' and reverse, 5'-AGGCTCGATGCTGTTCATCT-3' for GLUT3; forward, 5'-CGTACCGCTTCCTTAGA-3' and reverse, 5'-GATGACACGAGGCTCAC-3' for ENO1; forward, 5'-CGAGATCCCTCCAAAATCAA-3' and reverse, 5'-TGT GGTCATGAGTCCTTCCA-3' for GAPDH; forward, 5'-CCA GTTCATTCACATCATCAG-3' and reverse, 5'-CTTACACGA GGTCACATAGC-3' for HK2; and forward, 5'-TGGAGTGGA ATGAATGTTGC-3' and reverse, 5'-ATAGCCCAGGATGTG TAGCC-3' for $\mathrm{LDH} \alpha$.

PCR array. To analyze alterations in the expression of human nutrient/drug transporters in hypoxia, an Human Drug Transporters RT ${ }^{2}$ Profiler ${ }^{\mathrm{TM}}$ PCR Array kit (Qiagen $\mathrm{GmbH}$ ) was used. Cells were seeded in $60 \mathrm{~mm}$ dishes at a density of $2.5 \times 10^{5}$ cells/dish in $\alpha$-MEM medium, and total RNA was isolated with an RNeasy Mini kit (Qiagen $\mathrm{GmbH}$ ) after $4 \mathrm{~h}$ of hypoxic induction. cDNA was synthesized using Reverse Transcription system (Promega Corporation), and gene expression was measured using the PCR array kit according to the manufacturer's protocols.

Lactate production assay. Lactate production was determined using an L-lactate colorimetric assay kit (cat. no. ab65331; Abcam) according to the manufacturer's protocols. The lactate standard and samples with assay buffer were prepared in 96-well plates, and then the reaction mix and lactate enzyme mix were added to each well. The reaction mixture was incubated for $30 \mathrm{~min}$ at room temperature and optical density (OD) values at a wavelength of $450 \mathrm{~nm}$ were measured with a microplate reader (Tecan Group AG., Männedorf, Switzerland). All lactate production measurements were measured in triplicate.

Glucose uptake assay. Glucose uptake was measured using a glucose uptake colorimetric assay kit (cat. no. ab-136955; Abcam) according to the manufacturer's protocols. Cells were washed and resuspended in Krebs-Ringer-Phosphate-Hepes

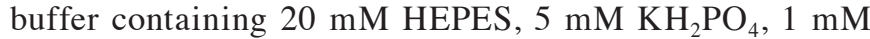
$\mathrm{MgSO}_{4}, 1 \mathrm{mM} \mathrm{CaCl}_{2}, 136 \mathrm{mM} \mathrm{NaCl}$ and $4.7 \mathrm{mM} \mathrm{KCl}$, $\mathrm{pH}$ 7.4. Glucose standards and samples with assay buffer were prepared in 96-well plates, and the enzyme and amplification reaction mixture was added to each well. The reaction solution was incubated for $30 \mathrm{~min}$ at $37^{\circ} \mathrm{C}$, and $\mathrm{OD}$ values at a wavelength of $412 \mathrm{~nm}$ were measured with a microplate reader (Tecan Group AG). All glucose uptake measurements were performed in triplicate.

Statistical analysis. Means and standard deviations were analyzed using Microsoft Excel software. Comparisons between two groups were conducted using Student's t-test. $\mathrm{P}<0.01$ was considered to indicate a statistically significant difference.

\section{Results}

$2 D$ proteomic analysis under hypoxia. 2D gel electrophoresis for proteomic analysis was performed. Hypoxia $(1 \%)$ increased the expression of 19 proteins ( $>2$-fold) and decreased the expression of 41 proteins ( $<2$-fold) as demonstrated by Coomassie Brilliant Blue staining (Fig. 1A). The 19 upregulated proteins were identified using LC-MS/MS and it was observed that the following proteins were upregulated under hypoxia (Table I): collagen $\alpha-1$ (I) chain preproprotein; chain A, moesin FERM domain bound to Ebp50 C-terminal peptide; chain $\mathrm{C}$, structure of UDP-glucose dehydrogenase V132 deletion; dystrophia myotonica-protein kinase isoform $\mathrm{B}$, partial; HLA-B associated transcript 1 variant; GDP dissociation inhibitor 2, isoform CRA-a; ENO1; chain C, ornithine aminotransferase mutant Y85i; proteasome (prosome, macropain) $26 \mathrm{~S}$ subunit, non-ATPase, 13 , isoform CRA-a; NADH dehydrogenase (ubiquinone) $1 \alpha$ subcomplex subunit 10 , mitochondrial; GAPDH; guanine nucleotide-binding protein subunit $\beta$-2-like 1; Rho GDP-dissociation inhibitor 1; trabeculin- $\alpha$; peroxiredoxin 1 , isoform CRA-b, partial; and peptidylprolyl isomerase A. However, the 42 downregulated were not identified. Of the upregulated proteins, ENO1 (499; Fig. 1A) and GAPDH (679; Fig. 1A) are directly involved in glycolysis, and their upregulation was examined using western blotting. As expected, it was observed that $1 \%$ hypoxia upregulated the protein expression levels of GAPDH and ENO1 in ASCs (Fig. 1B; after 8 and $16 \mathrm{~h}$ under hypoxia).

Hypoxia induces lactate production and glycolytic enzyme expression in ASCs. As ENO1 and GAPDH protein expression were significantly upregulated under hypoxia, lactate production was also measured. Hypoxia significantly increased lactate production by ASCs at $16 \mathrm{~h}$ (Fig. $2 \mathrm{~A}$; P<0.01). In addition, the mRNA expression levels of other glycolytic enzymes in ASCs were measured. As presented in Fig. 2B, hypoxia for 8 and 16 h significantly increased the mRNA expression levels of the GAPDH, ENO1, HK2 and LDH $\alpha$ genes as determined by qPCR analysis $(\mathrm{P}<0.01)$.

DFX $(50 \mu \mathrm{M})$, a pharmacological mimic of hypoxia, also significantly increased lactate production by ASCs at $16 \mathrm{~h}$ (Fig. 3A; $\mathrm{P}<0.01$ ). Similarly to $1 \%$ hypoxia, treatment with chemical hypoxia (at 8 and $16 \mathrm{~h}$ ) significantly upregulated mRNA expression levels of GAPDH, ENO1, HK2, and LDH $\alpha$ genes in ASCs (Fig. 3B; P<0.01). 
Table I. List of upregulated proteins identified in the two-dimensional proteomic screen.

\begin{tabular}{|c|c|c|c|c|}
\hline $\begin{array}{l}\text { Spot } \\
\text { no. }\end{array}$ & $\begin{array}{l}\text { Protein name } \\
\quad(P<0.05)\end{array}$ & $\begin{array}{c}\text { Normoxia } \\
\text { (\% vol) }\end{array}$ & $\begin{array}{l}\text { Hypoxia } \\
\text { (\% vol) }\end{array}$ & $\begin{array}{l}\mathrm{H} / \mathrm{N} \\
\text { ratio }\end{array}$ \\
\hline 54 & Collagen alpha-1(I) chain preproprotein & 0.038 & 0.078 & 2.0 \\
\hline 220 & Chain A, moesin FERM domain bound to Ebp50 C-terminal peptide & 0.079 & 0.175 & 2.2 \\
\hline 384 & Chain C, structure of UDP-glucose dehydrogenase V132 deletion & 0.070 & 0.137 & 2.0 \\
\hline 460 & Dystrophia myotonica-protein kinase isoform B, partial & 0.018 & 0.038 & 2.1 \\
\hline 471 & HLA-B associated transcript 1 variant & 0.025 & 0.049 & 2.0 \\
\hline 483 & GDP dissociation inhibitor 2, isoform CRA_a & 0.039 & 0.081 & 2.0 \\
\hline 499 & Enolase 1 & 0.021 & 0.077 & 3.7 \\
\hline 553 & Chain $\mathrm{C}$, ornithine aminotransferase mutant Y85i & 0.094 & 0.301 & 3.2 \\
\hline 629 & Proteasome (prosome, macropain) 26S subunit, non-ATPase, 13, isoform CRA_a & 0.034 & 0.098 & 2.9 \\
\hline 658 & NADH dehydrogenase (ubiquinone) $1 \alpha$ subcomplex subunit 10, mitochondrial & 0.048 & 0.094 & 2.0 \\
\hline 679 & Glyceraldehyde-3-phosphate dehydrogenase & 0.844 & 1.946 & 2.3 \\
\hline 791 & Guanine nucleotide-binding protein subunit $\beta$-2-like 1 & 0.128 & 0.310 & 2.4 \\
\hline 882 & Rho GDP-dissociation inhibitor 1 & 0.089 & 0.192 & 2.2 \\
\hline 975 & Trabeculin- $\alpha$ & 0.111 & 0.226 & 2.0 \\
\hline 1004 & Peroxiredoxin 1, isoform CRA_b, partial & 0.089 & 0.294 & 3.3 \\
\hline 1114 & Peptidylprolyl isomerase A (cyclophilin A) & 0.239 & 0.564 & 2.4 \\
\hline
\end{tabular}

$\mathrm{H} / \mathrm{N}$, hypoxia/normoxia.
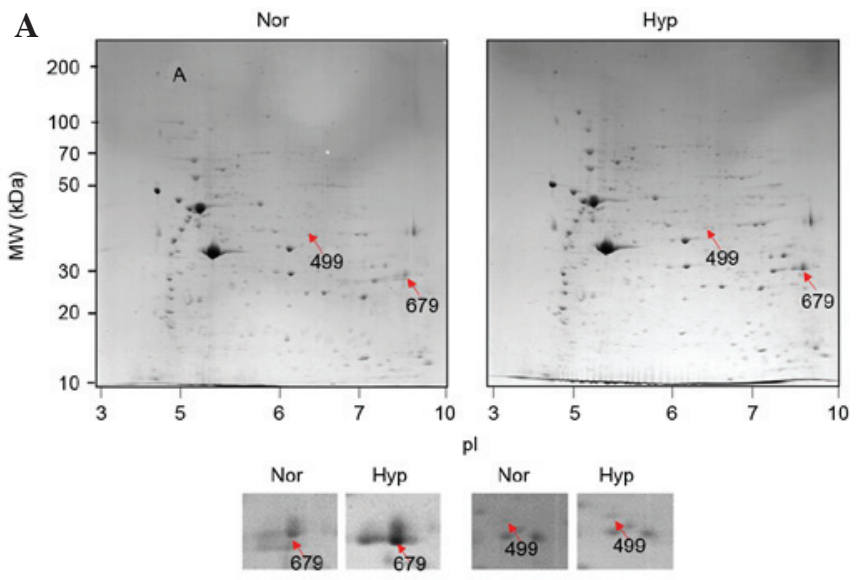

B

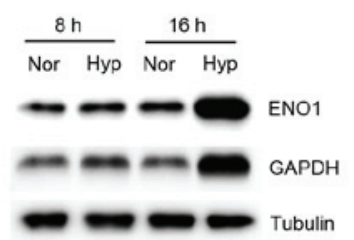

Figure 1. Proteomic analysis of ASCs under hypoxia. Two-dimensional gel electrophoresis $(n=1)$ was performed and upregulated proteins $(>2$ fold $)$ were identified. (A) Hypoxia (1\%) increased the expression of 19 proteins (>2-fold) as visualized by Coomassie blue staining. Of these proteins, spot 449 (ENO1) and spot 679 (GAPDH) were increased under hypoxia, and these proteins are involved in glycolysis. (B) In addition, hypoxia upregulated GAPDH and ENO1 at 8 and $16 \mathrm{~h}$, as determined by western blotting $(\mathrm{n}=1)$. Nor, normoxia; Hyp, hypoxia; ENO1, enolase 1; ASCs, adipose-derived stem cells.

HIF-1 $\alpha$ was involved in hypoxia-induced lactate production and glycolytic enzyme expression. As HIF-1 $\alpha$ has been reported to mediate the functional alterations of ASCs under hypoxia $(1,3,20)$, the current study measured HIF-1 $\alpha$ induction using western blotting. As expected, HIF-1 $\alpha$ was induced under hypoxia (Fig. 4A). Conversely, inhibitors of the HIF-1 $\alpha$ signaling pathway (50 $\mu \mathrm{M} \mathrm{YC}-1$ treatment) attenuated the induction of HIF-1 $\alpha$.

The involvement of the HIF-1 $\alpha$ signaling pathway in lactate production was further investigated using pharmacological inhibition studies. As expected, YC-1 (50 $\mu \mathrm{M})$ significantly attenuated hypoxia-induced lactate production at $16 \mathrm{~h}$ under hypoxia (Fig. 4B; $\mathrm{P}<0.01$ ). In addition, the altered mRNA expression levels of glycolytic enzymes were measured. YC-1 also attenuated the induction of mRNA expression of GAPDH, ENO1, HK2, and LDH $\alpha$ at $16 \mathrm{~h}$ under hypoxia in ASCs (Fig. 4C; $\mathrm{P}<0.01$ ).

Hypoxia increases glucose uptake in ASCs. As glycolysis pathways are induced under hypoxia, the present study examined whether or not hypoxia increases glucose uptake in ASCs. The altered expression of nutrient/drug transporters in ASCs was examined using an $\mathrm{RT}^{2}$ Profiler PCR array. Hypoxia significantly upregulated solute carrier family 2 (SLC2) member 1 (GLUT1) expression (data not shown). Thus, enhanced glucose uptake by ASCs was measured using a glucose uptake assay kit and it was demonstrated that $1 \%$ hypoxia significantly increased glucose uptake (Fig. 5A; $\mathrm{P}<0.01$ ). In addition to GLUT1, other SLC2 family members (the GLUT genes) have been reported to mediate glucose uptake in various cells $(21,22)$, and so alterations of expression of other SLC2 family members in ASCs was also examined. As presented in Fig. 5 and Table II, hypoxia significantly upregulated the mRNA expression levels of GLUT1 (Fig. 5B; $\mathrm{P}<0.01$ ), GLUT3 (Fig. 5C; P<0.01), and GLUT5 of ASCs as determined by qPCR. However, as GLUT5 predominantly mediates fructose transport, it was assumed that GLUT1 and 

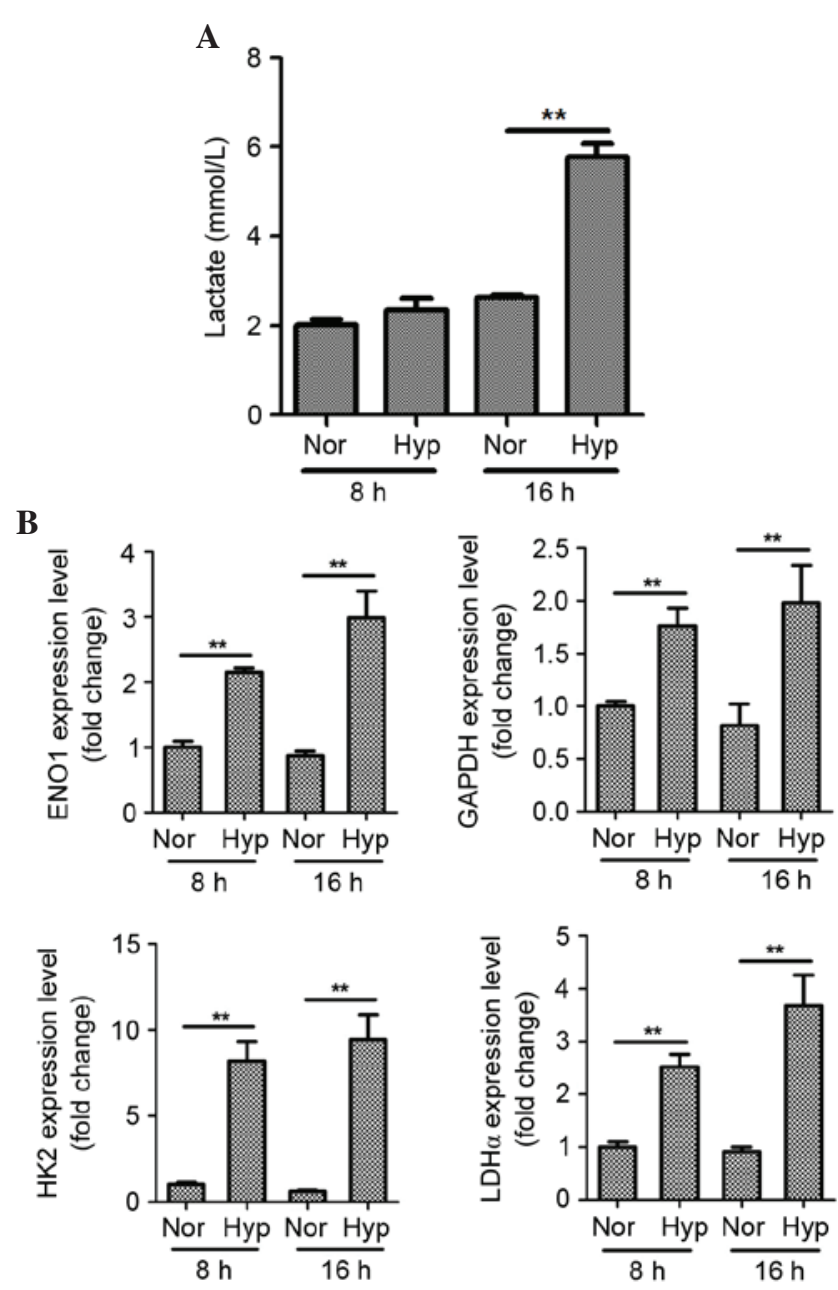

Figure 2. Hypoxia induces lactate production and expression of glycolytic enzyme mRNAs in ASCs. (A) Hypoxia (1\%) significantly increased lactate production by ASCs at $16 \mathrm{~h}$. (B) Hypoxia significantly increased the mRNA levels of GAPDH, ENO1, HK2, and LDH $\alpha$ at 8 and $16 \mathrm{~h}$ as determined by quantitative polymerase chain reaction analysis. $\mathrm{n}=3,{ }^{* *} \mathrm{P}<0.01$. Nor, normoxia; Hyp, hypoxia; ASCs, adipose-derived stem cells; ENO1, enolase 1; HK2, hexokinase 2; LDH $\alpha$, lactate dehydrogenase $\alpha$.

GLUT3 largely mediate hypoxia-increased glucose uptake in ASCs. The present study also confirmed the increased protein expression of GLUT1 and GLUT3 under hypoxia by western blotting (Fig. 5D).

HIF-1 $\alpha$ is involved in hypoxia-induced GLUT expression. It was further examined whether or not HIF-1 $\alpha$ mediates GLUT expression. As expected, YC-1 inhibited the expression of GLUT1 (Fig. 6A; P<0.01) and GLUT3 (Fig. 6B; P<0.01) under hypoxic conditions in ASCs. These results indicate that the HIF-1 $\alpha$ is involved in hypoxia-induced glucose uptake by ASCs.

\section{Discussion}

The present study primarily investigated the altered glucose uptake and metabolism of ASCs under hypoxia. In addition, the current study aimed to identify the underlying molecular mechanisms involved in the hypoxia-induced glucose uptake and lactate production of ASCs. As determined by proteomic analysis and western blotting, GAPDH and ENO1 expression levels were upregulated under hypoxia. In addition, lactate

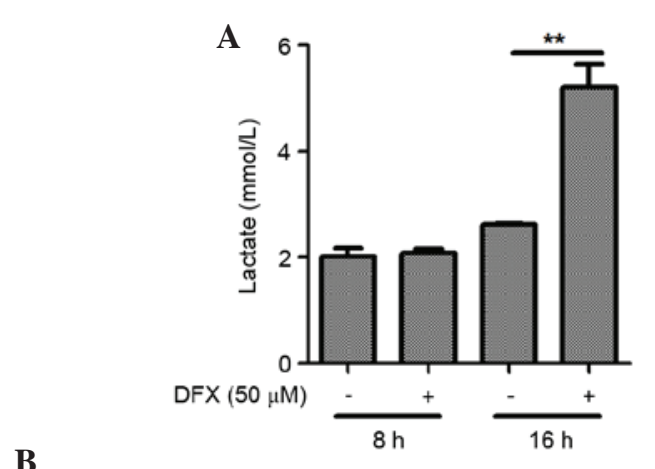

B
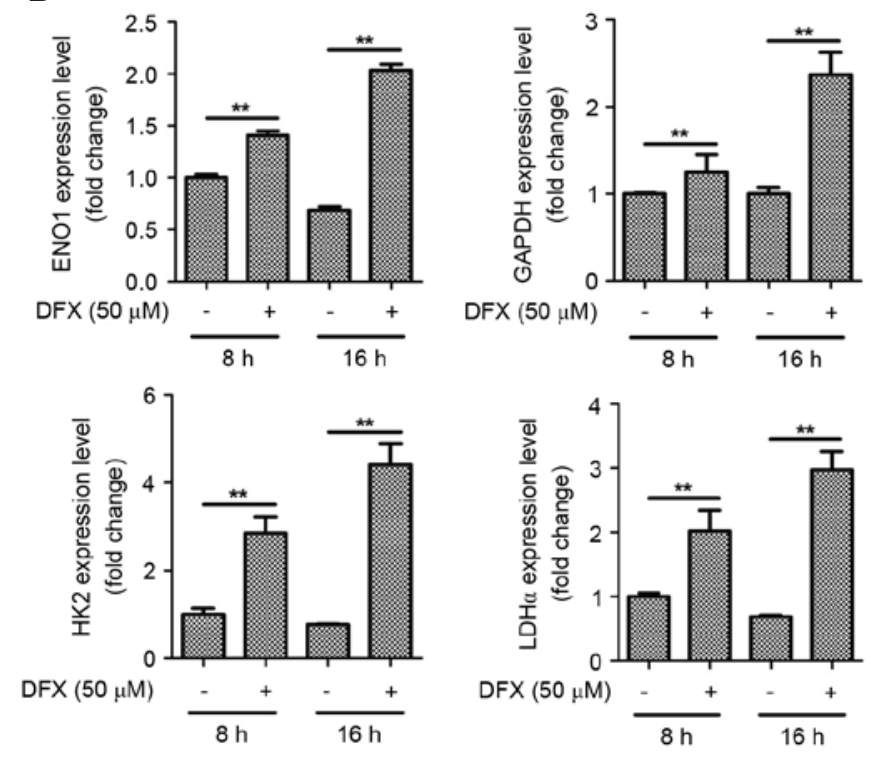

Figure 3. Hypoxia mimic also induces lactate production and mRNA expression of glycolytic enzymes in ASCs. (A) DFX $(50 \mu \mathrm{M})$, a pharmacological mimetic of hypoxia, increased lactate production by ASCs. (B) DFX also upregulated the mRNA expression of GAPDH, ENO1, HK2, and LDH $\alpha$ in ASCs. $n=3,{ }^{* *} \mathrm{P}<0.01$. DFX, deferoxamine; ASCs, adipose-derived stem cells; ENO1, enolase 1; HK2, hexokinase 2; LDH $\alpha$, lactate dehydrogenase $\alpha$.

production was significantly increased, and the mRNA levels of glycolytic enzymes were elevated. The underlying mechanism involved in the upregulation of these genes was investigated, and it was observed that HIF-1 $\alpha$ protein expression levels were increased. Pharmacological inhibitors of HIF-1 $\alpha$ significantly attenuate hypoxia-induced lactate production and glycolytic enzyme expression. As glycolytic pathways are induced under hypoxia, the present study investigated whether or not hypoxia increases glucose uptake in ASCs. As expected, hypoxia significantly increased glucose uptake in ASCs. The mRNA expression levels of glucose transporters were measured in ASCs under hypoxia, and it was demonstrated that GLUT1 and GLUT3 expression were upregulated, as determined by qPCR and western blotting. It was also observed that pharmacological inhibition of HIF-1 $\alpha$ significantly attenuated hypoxia-induced GLUT1 and GLUT3 expression. These results indicate that hypoxia increases glucose uptake via GLUT1 and GLUT3 upregulation, and induces the anaerobic metabolism of ASCs via GAPDH, ENO1, HK2 and LDH $\alpha$.

Proteomic analysis has been used to investigate the underlying molecular mechanisms of proliferation and differentiation of mesenchymal stem cells (MSCs). Using 
Table II. GLUT expression under hypoxic conditions.

\begin{tabular}{lcccr}
\hline $\begin{array}{l}\text { GLUT } \\
\text { isoform }\end{array}$ & $\begin{array}{c}\text { Normoxia } 8 \mathrm{~h} \\
(\mathrm{Cq} \pm \mathrm{SD})\end{array}$ & $\begin{array}{c}\text { Hypoxia } 8 \mathrm{~h} \\
(\mathrm{Cq} \pm \mathrm{SD})\end{array}$ & $\begin{array}{c}\text { Normoxia } 16 \mathrm{~h} \\
(\mathrm{Cq} \pm \mathrm{SD})\end{array}$ & $\begin{array}{c}\text { Hypoxia } 16 \mathrm{~h} \\
(\mathrm{Cq} \pm \mathrm{SD})\end{array}$ \\
\hline Glut1 & $27.82 \pm 0.19$ & $26.23 \pm 0.20$ & $28.59 \pm 0.31$ & $25.98 \pm 0.03$ \\
Glut2 & $28.74 \pm 0.2$ & $29.59 \pm 0.15$ & $29.87 \pm 0.32$ & $30.1 \pm 0.27$ \\
Glut3 & $24.3 \pm 0.11$ & $23.36 \pm 0.09$ & $24.52 \pm .14$ & $23.12 \pm 0.04$ \\
Glut4 & $30.77 \pm 0.06$ & $31.2 \pm 0.28$ & $31.4 \pm 0.43$ & $31.98 \pm 0.18$ \\
Glut5 & $30.62 \pm 0.25$ & $28.71 \pm 0.22$ & $30.81 \pm 0.13$ & $26.65 \pm 0.13$ \\
Glut8 & $27.04 \pm 0.01$ & $27.35 \pm 0.1$ & $27.09 \pm 0.15$ & $27.05 \pm 0.03$ \\
Glut12 & $28.92 \pm 0.11$ & $29.6 \pm 0.03$ & $29.5 \pm 0.1$ & $29.68 \pm 0.08$ \\
\hline
\end{tabular}

GLUT, glucose transporter; Cq, cycle quantification; SD, standard deviation.

A

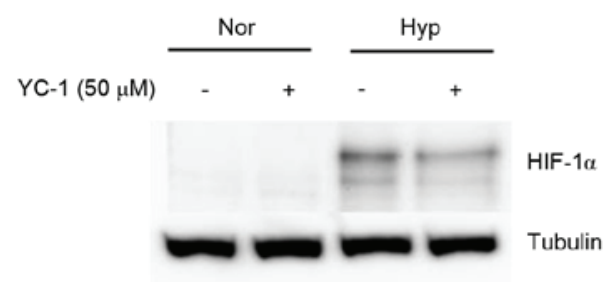

C

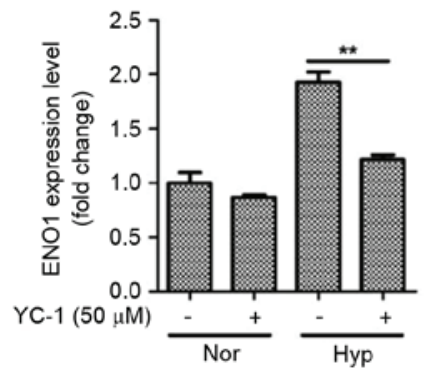

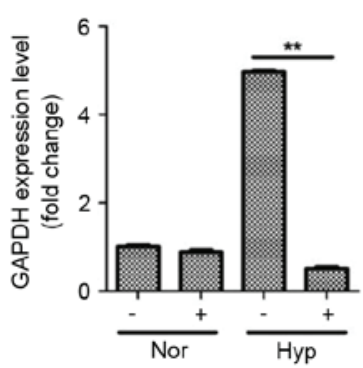
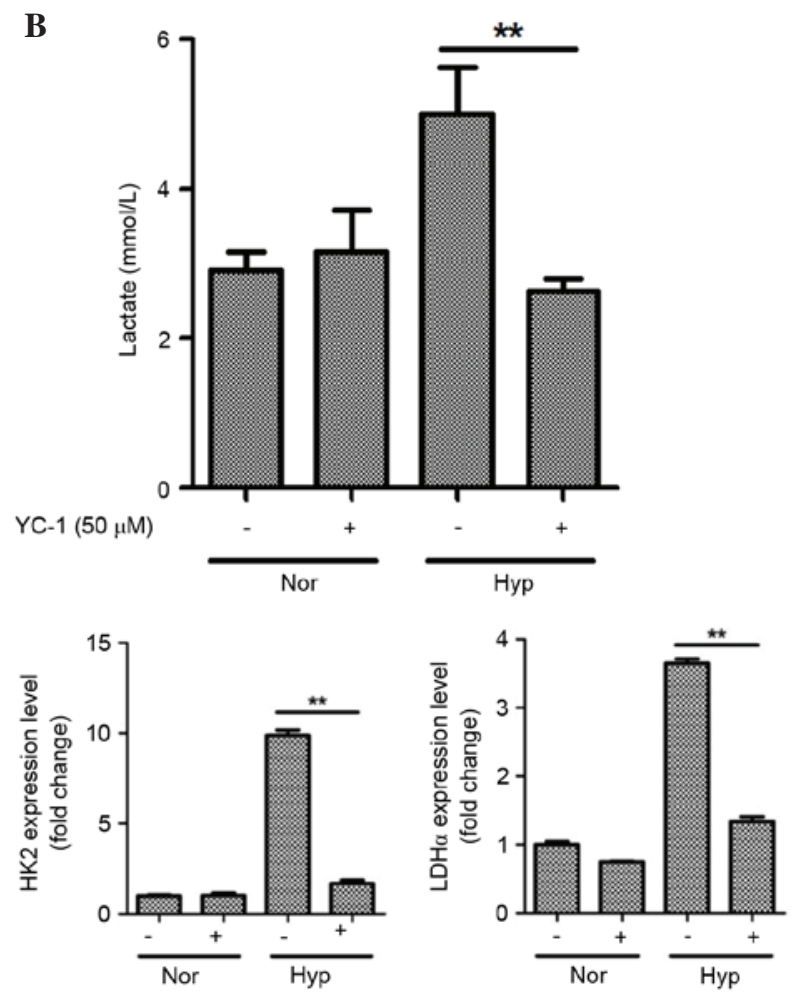

Figure 4. HIF-1 $\alpha$ is involved in lactate production and mRNA expression of glycolytic enzymes in ASCs. (A) Hypoxia (1\%) stabilized the HIF-1 $\alpha$ expression, however, YC-1 reduced the HIF-1 $\alpha$ protein expression levels as demonstrated by western blotting. (B) YC-1 inhibited lactate production under hypoxia. (C) YC-1 also attenuated the hypoxia-induced mRNA level of GAPDH, ENO1, HK2, and LDH $\alpha$ at 16 h in ASCs. n=3, ${ }^{* *}$ P $<0.01$. Nor, normoxia; Hyp, hypoxia; HIF-1 $\alpha$, hypoxia-inducible factor 1- $\alpha$; ASCs, adipose-derived stem cells; ENO1, enolase 1; HK2, hexokinase 2; LDH $\alpha$, lactate dehydrogenase $\alpha$.

a 2D electrophoretic analysis, Roche et al (23) reported that the intracellular proteins of bone marrow-derived MSCs and ASCs are similar, and that these two cell types are interchangeable with respect to use in cell-based therapy. A comparative secretome analysis of MSCs demonstrated that during osteogenesis, calcium homeostasis-associated proteins are upregulated, whereas, stem cell proliferation-associated proteins and other lineage-associated proteins are downregulated (24). Zeng et al (25) investigated the effects of chemical hypoxia induced by cobalt chloride on human umbilical cord MSCs and observed that peroxiredoxin 1 was downregulated, whereas, ENO1 and synaptic vesicle membrane protein VAT-1 homolog were upregulated. The present study examined glucose uptake and metabolism under hypoxia using 2D proteomic analysis, and observed that physical hypoxia (1\% oxygen) significantly upregulated GAPDH and ENO1 expression to induce the glycolytic pathway in ASCs.

GAPDH is regarded as a housekeeping gene, and is widely used as a reference gene in RT-PCR and qPCR. Accordingly, the mRNA expression levels of certain growth factors were measured under hypoxia and their expression was compared using GAPDH as a reference gene. Although the cycle of quantification value of GAPDH under hypoxia was not different from that under normoxia in previous studies $(2 \%$ oxygen, serum-free, at $4 \mathrm{~h})(4,8), 1 \%$ hypoxia in fetal bovine serum (usually at $16 \mathrm{~h}$ ) in the present study significantly increased 
A
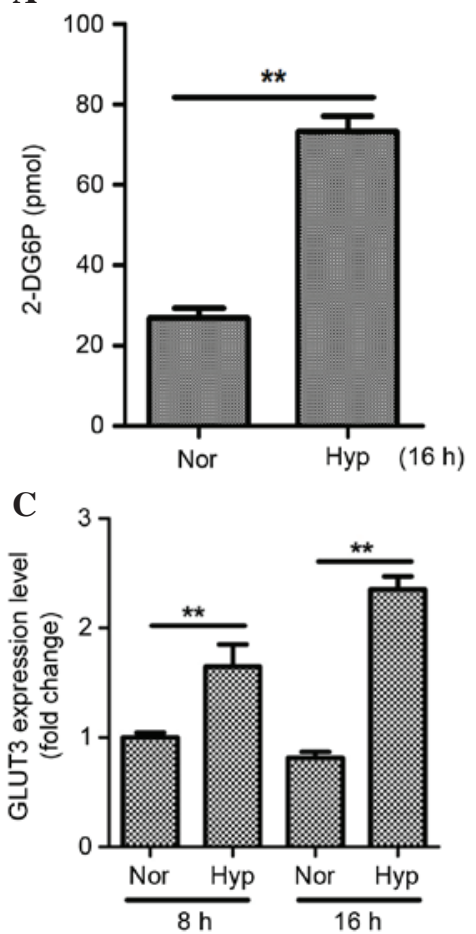

B

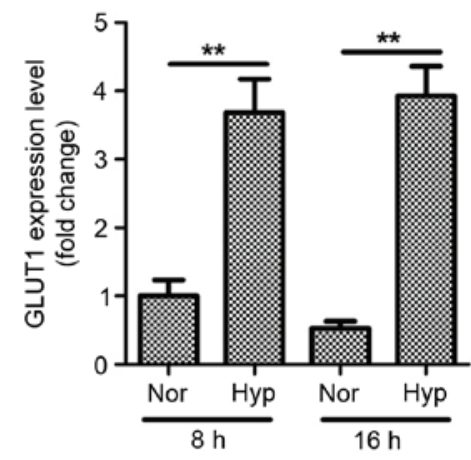

D

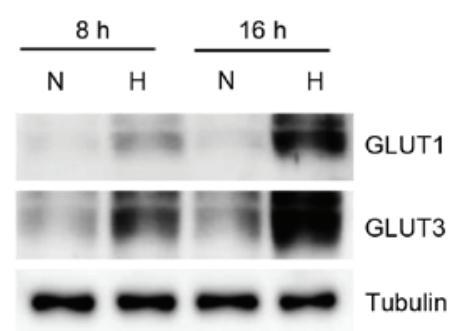

Figure 5. Hypoxia increases glucose uptake in ASCs. (A) Hypoxia (1\%) significantly increased glucose uptake at $16 \mathrm{~h}$, as determined by glucose uptake assay $(\mathrm{n}=3)$. Hypoxia significantly upregulated the mRNA level of (B) GLUT1 and (C) GLUT3 of ASCs, as determined by quantitative polymerase chain reaction $(\mathrm{n}=3)$. (D) Hypoxia also increased protein expression levels of GLUT1 and GLUT3, as determined by western blotting. ${ }^{* *} \mathrm{P}<0.01$. ASCs, adipose-derived stem cells; GLUT, glucose transporter; 2-DG6P, 2-deoxy-glucose-6-phosphate; Nor, normoxia; Hyp, hypoxia.
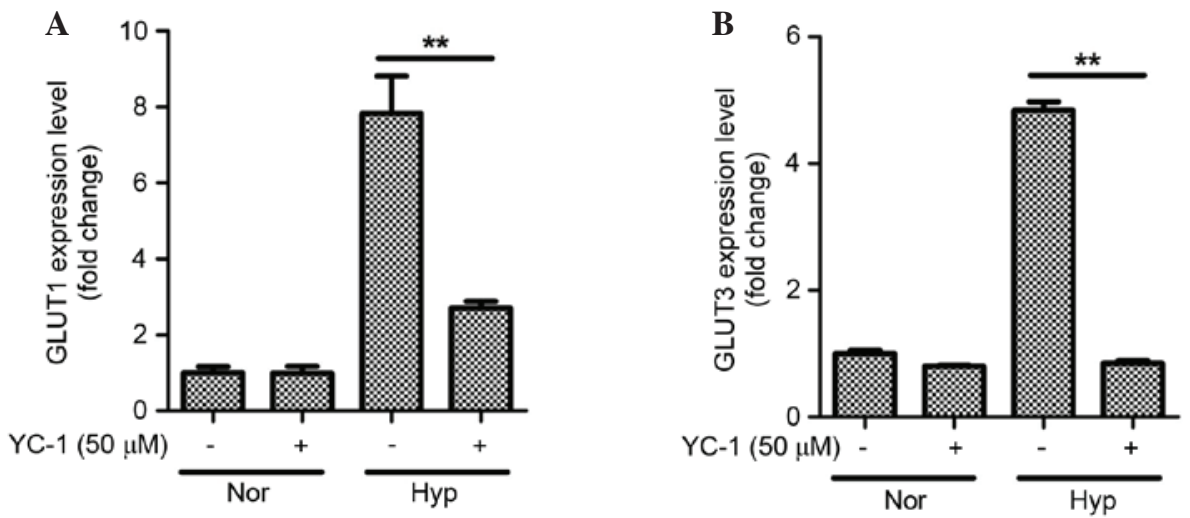

Figure 6. Hypoxia-inducible factor 1- $\alpha$ involvement in the hypoxia-induced GLUT expression. YC-1 inhibited the expression of (A) GLUT1 and (B) GLUT3 in adipose-derived stem cells. $\mathrm{n}=3,{ }^{* *} \mathrm{P}<0.01$. GLUT, glucose transporter; Nor, normoxia; Hyp, hypoxia.

the expression level of GAPDH mRNA in ASCs. Although GAPDH has long been used as a default reference gene in quantitative mRNA profiling experiments, its expression reportedly varies in response to HIF- $1 \alpha$ in a range of pathophysiological environments, including inflammation, oxidative stress, hyperinsulinemia and hypoxia (26-28). Thus, GAPDH is an unreliable housekeeping gene for normalizing gene expression under hypoxia, and should be replaced by alternative validated reference genes $(26,28)$. GAPDH should be used with caution as a reference gene in hypoxia-associated experiments.

A preliminary study investigated the altered expression of nutrient/drug transporters under hypoxia using an $\mathrm{RT}^{2}$ Profiler PCR array. In addition to its effect on SLC2A1 (GLUT1) mRNA levels, $1 \%$ hypoxia increased solute carrier family
22 member 1 (SLC22A1; also termed organic cation transporter 1) mRNA expression levels (2-fold increase). However, SLC22A1 expression did not change in qPCR analysis in a preliminary study. Although nutrient/drug transporters may have diverse effects on ASCs, their physiological or functional roles have not been fully investigated. We previously demonstrated that vitamin $\mathrm{C}$ treatment increases the proliferation of and enhances the hair-regenerative potential of ASCs via a sodium-dependent vitamin C transporter (6). The present study demonstrated that hypoxia upregulated GLUT1 and GLUT3 expression, which increased glucose uptake by ASCs, thereby inducing their proliferation. van Dijk et al (29) also reported that breast cancer resistance protein protects ASCs against ischemic damage. Although studies regarding nutrient/drug 
transporters in stem cell research are in their early stages, the functional role of nutrient/drug transporters in ASCs may be notable.

GLUT is a membrane protein that facilitates the transport of glucose across cell membranes. GLUT contains 12 membrane-spanning helices, and, to date, 13 members of the GLUT/SLC2 family have been identified (30-32). Tissue expression, substrate specificity, and transport kinetics of GLUT differ in physiological conditions. Notably, hypoxia has been reported to alter the expression of specific isoforms of GLUT, and also to alter glucose metabolism in various types of cell (33). For example, the hypoxia-responsive proteins GLUT1 and GLUT3 function as glucose sensors in chondrocytes, and their expression levels are upregulated by HIF-1 $\alpha$ (33). Hypoxic preconditioning also stimulates glucose uptake and metabolism of rat bone marrow-derived MSCs, via upregulation of the expression of HIF-1 $\alpha$ and GLUT1 (34). In addition to hypoxia, bFGF also induces HIF-1 $\alpha$, and regulates glucose metabolism via GLUT1 induction in adipocytes (35). The present study demonstrated that hypoxia significantly induces GLUT1 and GLUT3 to increase the proliferation of ASCs.

In conclusion, the current study investigated the altered glucose uptake and metabolism of ASCs under hypoxia, and identified the underlying signaling pathways and molecular mechanisms. The results of the present study indicate that hypoxia increases glucose uptake via GLUT1 and GLUT3 upregulation, and induces lactate production via GAPDH, ENO1, HK2 and LDH $\alpha$. Furthermore, HIF-1 $\alpha$ signaling pathways are involved in this altered glucose uptake and metabolism in ASCs. Thus, anaerobic metabolism under hypoxia favors the proliferation of ASCs.

\section{Acknowledgements}

The present study was supported by the National Research Foundation, funded by the Korean government (grant no. 2014054836) and Chung-Ang University Research Grants in 2015.

\section{References}

1. Chung HM, Won CH and Sung JH: Responses of adipose-derived stem cells during hypoxia: Enhanced skin-regenerative potential. Expert Opin Biol Ther 9: 1499-1508, 2009.

2. Kim JH, Park SH, Park SG, Choi JS, Xia Y and Sung JH: The pivotal role of reactive oxygen species generation in the hypoxia-induced stimulation of adipose-derived stem cells. Stem Cells Dev 20: 1753-1761, 2011.

3. Lee EY, Xia Y, Kim WS, Kim MH, Kim TH, Kim KJ, Park BS and Sung JH: Hypoxia-enhanced wound-healing function of adipose-derived stem cells: Increase in stem cell proliferation and up-regulation of VEGF and bFGF. Wound Repair Regen 17: 540-547, 2009

4. Kim JH, Song SY, Park SG, Song SU, Xia Y and Sung JH: Primary involvement of NADPH oxidase 4 in hypoxia-induced generation of reactive oxygen species in adipose-derived stem cells. Stem Cells Dev 21: 2212-2221, 2012.

5. Kang S, Kim SM and Sung JH: Cellular and molecular stimulation of adipose-derived stem cells under hypoxia. Cell Biol Int 38: 553-562, 2014.

6. Kim JH, Kim WK, Sung YK, Kwack MH, Song SY, Choi JS, Park SG, Yi T, Lee HJ, Kim DD, et al: The molecular mechanism underlying the proliferating and preconditioning effect of vitamin C on adipose-derived stem cells. Stem Cells Dev 23: $1364-1376,2014$
7. Hye Kim J, Gyu Park S, Kim WK, Song SU and Sung JH: Functional regulation of adipose-derived stem cells by PDGF-D. Stem Cells 33: 542-556, 2015.

8. Kim JH, Park SG, Song SY, Kim JK and Sung JH: Reactive oxygen species-responsive miR-210 regulates proliferation and migration of adipose-derived stem cells via PTPN2. Cell Death Dis 4: e588, 2013.

9. Park BS, Kim WS, Choi JS, Kim HK, Won JH, Ohkubo F and Fukuoka H: Hair growth stimulated by conditioned medium of adipose-derived stem cells is enhanced by hypoxia: Evidence of increased growth factor secretion. Biomed Res 31: 27-34, 2010.

10. Kim JH, Kim SH, Song SY, Kim WS, Song SU, Yi T, Jeon MS, Chung HM, Xia Y and Sung JH: Hypoxia induces adipocyte differentiation of adipose-derived stem cells by triggering reactive oxygen species generation. Cell Biol Int 38: 32-40, 2014.

11. Hsu SH, Chen CT and Wei YH: Inhibitory effects of hypoxia on metabolic switch and osteogenic differentiation of human mesenchymal stem cells. Stem cells 31: 2779-2788, 2013.

12. Meretoja VV, Dahlin RL, Wright S, Kasper FK and Mikos AG: The effect of hypoxia on the chondrogenic differentiation of co-cultured articular chondrocytes and mesenchymal stem cells in scaffolds. Biomaterials 34: 4266-4273, 2013.

13. Gaspar JA, Doss MX, Hengstler JG, Cadenas C, Hescheler J and Sachinidis A: Unique metabolic features of stem cells, cardiomyocytes, and their progenitors. Circ Res 114: 1346-1360, 2014.

14. Ito $\mathrm{K}$ and Suda T: Metabolic requirements for the maintenance of self-renewing stem cells. Nat Rev Mol Cell Biol 15: 243-256, 2014.

15. Dong Q, Yang Y, Song L, Qian H and Xu Z: Atorvastatin prevents mesenchymal stem cells from hypoxia and serum-free injury through activating AMP-activated protein kinase. Int J Cardiol 153: 311-316, 2011.

16. Suda T, Takubo K and Semenza GL: Metabolic regulation of hematopoietic stem cells in the hypoxic niche. Cell Stem Cell 9: 298-310, 2011.

17. Mohyeldin A, Garzon-Muvdi T and Quinones-Hinojosa A: Oxygen in stem cell biology: A critical component of the stem cell niche. Cell Stem Cell 7: 150-161, 2010.

18. Buravkova LB, Rylova YV, Andreeva ER, Kulikov AV, Pogodina MV, Zhivotovsky B and Gogvadze V: Low ATP level is sufficient to maintain the uncommitted state of multipotent mesenchymal stem cells. Biochim Biophys Acta 1830: 4418-4425, 2013

19. Yi T, Kim WK, Choi JS, Song SY, Han J, Kim JH, Kim WS, Park SG, Lee HJ, Cho YK, et al: Isolation of adipose-derived stem cells by using a subfractionation culturing method. Expert Opin Biol Ther 14: 1551-1560, 2014.

20. Gao W, Qiao X, Ma S and Cui L: Adipose-derived stem cells accelerate neovascularization in ischaemic diabetic skin flap via expression of hypoxia-inducible factor- $1 \alpha$. J Cell Mol Med 15: 2575-2585, 2011.

21. Ren BF, Deng LF, Wang J, Zhu YP, Wei L and Zhou Q: Hypoxia regulation of facilitated glucose transporter-1 and glucose transporter-3 in mouse chondrocytes mediated by HIF-1alpha. Joint Bone Spine 75: 176-181, 2008.

22. Vannucci SJ, Reinhart R, Maher F, Bondy CA, Lee WH, Vannucci RC and Simpson IA: Alterations in GLUT1 and GLUT3 glucose transporter gene expression following unilateral hypoxia-ischemia in the immature rat brain. Brain Res Dev Brain Res 107: 255-264, 1998.

23. Roche S, Delorme B, Oostendorp RA, Barbet R, Caton D, Noel D, Boumediene K, Papadaki HA, Cousin B, Crozet C, et al: Comparative proteomic analysis of human mesenchymal and embryonic stem cells: Towards the definition of a mesenchymal stem cell proteomic signature. Proteomics 9: 223-232, 2009.

24. Kim JM, Kim J, Kim YH, Kim KT, Ryu SH, Lee TG and Suh PG: Comparative secretome analysis of human bone marrow-derived mesenchymal stem cells during osteogenesis. J Cell Physiol 228: 216-224, 2013

25. Zeng HL, Zhong Q, Jia HT, Qing YL, Bu QQ, Han XA and Liu HW: Differential proteomic analysis in human umbilical cord mesenchymal stem cells induced by cobalt chloride. Zhonghua Xue Ye Xue Za Zhi 32: 739-743, 2011 (In Chinese).

26. Tan SC, Carr CA, Yeoh KK, Schofield CJ, Davies KE and Clarke K: Identification of valid housekeeping genes for quantitative RT-PCR analysis of cardiosphere-derived cells preconditioned under hypoxia or with prolyl-4-hydroxylase inhibitors. Mol Biol Rep 39: 4857-4867, 2012. 
27. Chiche J, Pommier S, Beneteau M, Mondragón L, Meynet O, Zunino B, Mouchotte A, Verhoeyen E, Guyot M, Pagès G, et al: GAPDH enhances the aggressiveness and the vascularization of non-Hodgkin's B lymphomas via NF-kB-dependent induction of

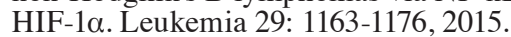

28. Cummings M, Sarveswaran J, Homer-Vanniasinkam S, Burke D and Orsi NM: Glyceraldehyde-3-phosphate dehydrogenase is an inappropriate housekeeping gene for normalising gene expression in sepsis. Inflammation 37: 1889-1894, 2014.

29. van Dijk A, Naaijkens BA, Jurgens WJ, Oerlemans R, Scheffer GL, Kassies J, Aznou J, Brouwer M, van Rossum AC, Schuurhuis GJ, et al: The multidrug resistance protein breast cancer resistance protein (BCRP) protects adipose-derived stem cells against ischemic damage. Cell Biol Toxicol 28: 303-315, 2012.

30. Gould GW and Holman GD: The glucose transporter family: Structure, function and tissue-specific expression. Biochem J 295: 329-341, 1993.

31. Mueckler M: Family of glucose-transporter genes. Implications for glucose homeostasis and diabetes. Diabetes 39: 6-11, 1990.
32. Olson AL and Pessin JE: Structure, function, and regulation of the mammalian facilitative glucose transporter gene family. Annu Rev Nutr 16: 235-256, 1996.

33. Mobasheri A, Richardson S, Mobasheri R, Shakibaei M and Hoyland JA: Hypoxia inducible factor- 1 and facilitative glucose transporters GLUT1 and GLUT3: Putative molecular components of the oxygen and glucose sensing apparatus in articular chondrocytes. Histol Histopathol 20: 1327-1338, 2005.

34. Zhu H, Chen X and Deng L: Effects of hypoxic preconditioning on glucose metabolism of rat bone marrow mesenchymal stem cells. Zhongguo Xiu Fu Chong Jian Wai Ke Za Zhi 25: 1004-1007, 2011 (In Chinese).

35. Kihira Y, Yamano N, Izawa-Ishizawa Y, Ishizawa K, Ikeda Y, Tsuchiya K, Tamaki T and Tomita S: Basic fibroblast growth factor regulates glucose metabolism through glucose transporter 1 induced by hypoxia-inducible factor-1alpha in adipocytes. Int J Biochem Cell Biol 43: 1602-1611, 2011. 